\title{
Excitons and Recombination in Photovoltaic Materials
}

S. Smith, H.M. Cheong, B.D. Fluegel

J.F. Geisz, J.V. Olson, R. Dhere, L.L. Kazmerski, and A. Mascarenhas

National Renewable Energy Laboratory

Presented at the National Center for

Photovoltaics Program Review Meeting

Denver, Colorado

September 8-11, 1998

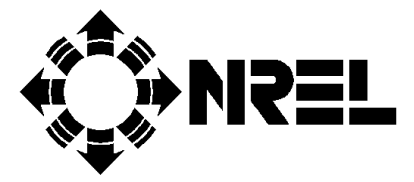

National Renewable Energy Laboratory 1617 Cole Boulevard

Golden, Colorado 80401-3393

A national laboratory of the U.S. Department of Energy

Managed by Midwest Research Institute

for the U.S. Department of Energy

under contract No. DE-AC36-83CH10093

Work performed under task number PV902201

October 1998 


\section{NOTICE}

This report was prepared as an account of work sponsored by an agency of the United States government. Neither the United States government nor any agency thereof, nor any of their employees, makes any warranty, express or implied, or assumes any legal liability or responsibility for the accuracy, completeness, or usefulness of any information, apparatus, product, or process disclosed, or represents that its use would not infringe privately owned rights. Reference herein to any specific commercial product, process, or service by trade name, trademark, manufacturer, or otherwise does not necessarily constitute or imply its endorsement, recommendation, or favoring by the United States government or any agency thereof. The views and opinions of authors expressed herein do not necessarily state or reflect those of the United States government or any agency thereof.

Available to DOE and DOE contractors from:

Office of Scientific and Technical Information (OSTI)

P.O. Box 62

Oak Ridge, TN 37831

Prices available by calling 423-576-8401

Available to the public from:

National Technical Information Service (NTIS)

U.S. Department of Commerce

5285 Port Royal Road

Springfield, VA 22161

703-605-6000 or 800-553-6847

or

DOE Information Bridge

http://www.doe.gov/bridge/home.html 


\title{
Excitons and Recombination in Photovoltaic Materials
}

\author{
S. Smith, H.M Cheong, B.D. Fluegel, J.F. Geisz, J.M. Olson, \\ R. Dhere, L.L. Kazmerski and A. Mascarenhas \\ National Renewable Energy Laboratory \\ Golden, Colorado 80401
}

\begin{abstract}
High spatial resolution $(\sim 0.7 \mu \mathrm{m})$ scanning confocal microscopy, combined with low-temperature (5K) photoluminescence (PL) spectroscopy, can be used to probe the spatial variations in the spectral properties of photovoltaic materials with submicron spatial resolution $(\sim 0.7 \mu \mathrm{m})$. We report on the successful demonstration of this technique applied to two particular photovoltaic systems: a partially ordered $\mathrm{GaInP}_{2}$ epilayer, and a released (exposing the CdTe/CdS interface) polycrystalline CdTe film.
\end{abstract}

\section{INTRODUCTION}

Emerging photovoltaic (PV) technologies are producing low-cost, high-efficiency solar cells using thin-film polycrystalline semiconductors. In these polycrystalline materials, grain size, chemical composition and doping can all vary between individual grains and across grain boundaries. So far, optical studies of these materials have relied on macroscopic techniques with probe sizes that are much larger than the average grain size. In these techniques, only average properties are measured. As a result, the current understanding regarding the microscopic fluctuations of the optoelectronic properties due to the grain structure, the role of grain boundaries as recombination centers, and passivation of the defect states near grain boundaries is very limited. By combining high spatial resolution scanning confocal microscopy with high resolution low-temperature PL spectroscopy, we have, for the first time to our knowledge, spatially resolved the PL emission in two such PV systems with sub-micron spatial resolution $(\sim 0.7 \mu \mathrm{m})$. The results and interpretation of these studies are discussed below.

\section{EXPERIMENTS}

The samples consisted of: 1) a $10 \mu \mathrm{m}$ thick GaInP $\mathrm{P}_{2}$ epilayer grown by organometallic vapor-phase epitaxy (OMVPE) at $680^{\circ} \mathrm{C}$ on a (001) semi-insulating 
GaAs substrate misoriented $6^{\circ}$ toward $[111]_{B}$, and 2) a CdTe film that was released from the substrate, directly exposing the CdS/CdTe interface (original structure: 5 $\mu \mathrm{m}$ CdTe grown by closed-space sublimation (CSS) at $610^{\circ} \mathrm{C} / 80 \mathrm{~nm} \mathrm{CdS} / 50 \mathrm{~nm}$ $\mathrm{SnO}_{2}$ on a borosilicate substrate, treated with $\mathrm{CdCl}_{2}$ at $400^{\circ} \mathrm{C}$ for $30 \mathrm{~min}$ ). Parts of sample 1 were patterned with aluminum masks containing small aperatures, which were used for the resolution tests discussed below. The samples were mounted on the stage of a commercially available low-temperature STM system (Oxford Instruments CryoSXM) which is retrofitted with a high numerical aperture microscope objective (Newport M-60X, NA=0.85) in place of the tunneling tip. Excitation from a green HeNe laser $(543 \mathrm{~nm})$ was coupled into a bifurcated single mode fiber and imaged confocally through the cryostat window onto the sample by the objective inside the cryostat $(\approx 1.7 \mu \mathrm{W}$ total power $)$. All focusing and sample positioning internal to the cryostat is piezoelectrically controlled via the STM systems inertial sliding mechanisms. The photoluminescence was imaged with the same optics in the reverse order, and the third output port of the bifurcated fiber was imaged onto the slit of a spectrometer/CCD where it is spectrally resolved with an energy resolution in this experiment of $\sim 40 \mu \mathrm{eV}$. All experiments were performed at $T=5.0 \mathrm{~K}$. Since the images are spectrally resolved, each image can be extruded in the spectral dimension, producing a series of images at every energy over the measured spectrum (2000 images, one for every pixel of the CCD used to image the spectrum).

In order to measure the spatial resolution, an aluminum mask patterned with a $10 \times 10$ array of $2500 \AA$ circular holes was formed on the surface of the same ordered $\mathrm{GaInP}_{2}$ sample which was to be studied (see reference [1] for details of the sample preparation). The PL intensity from one of these small holes was imaged with the above described system at $5.0 \mathrm{~K}$ and is displayed in the left half of Figure 1. The right half of Figure 1 shows a simulated image which is the result of convolving a gaussian point spread function (PSF) with a $2500 \AA$ circular disk (a noise level comparable to the measured image was added to the calculated image). The gaussian full-width at half-maximum (FWHM) which produces the best match to the measured image is $0.7 \mu \mathrm{m}$, from which we infer the spatial resolution of the instrument.

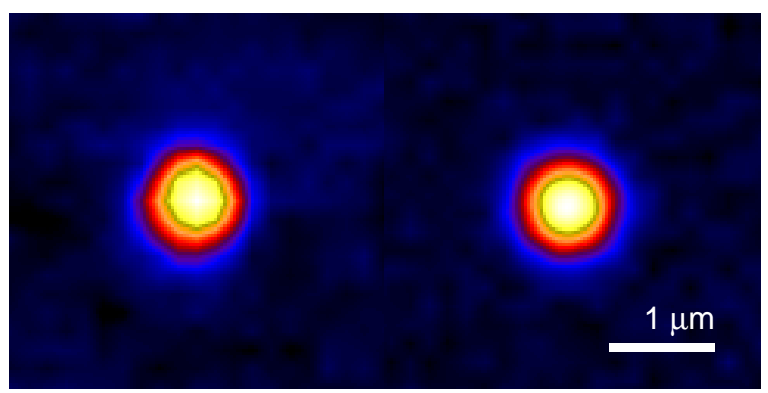

FIGURE 1. Measured (left) and calculated (right) images of $0.25 \mu \mathrm{m}$ aperture. 


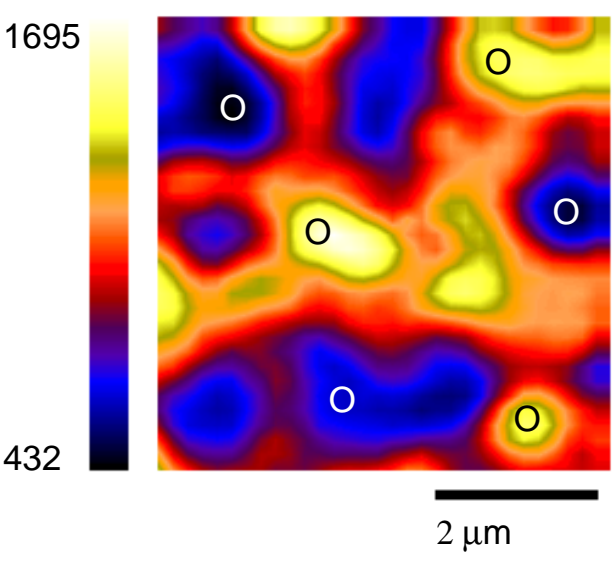

(a)

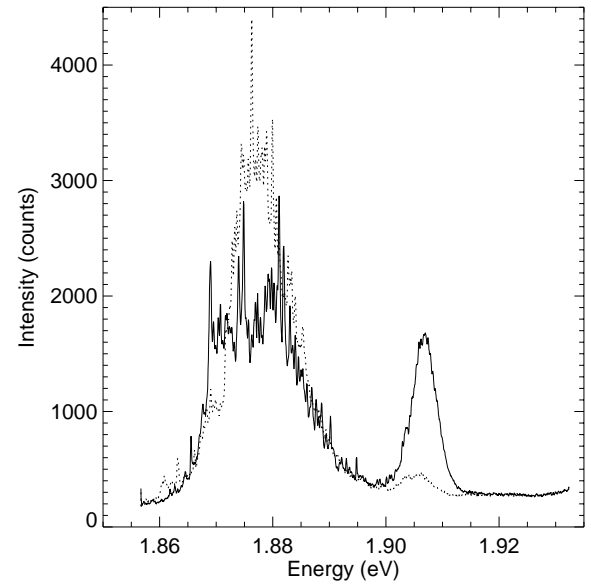

(b)

FIGURE 2. a): Spatial map of PL intensity at the average excitonic energy (scale in units of counts/second). Select areas where the excitonic peak is strong are marked by black circles, areas where exitonic peak is suppressed are marked by white circles (see text). b): Spectra taken at locations marked by the leftmost black (solid line) and white (dotted line) circles (overlaid).

Results from GaInP $P_{2}$ sample: Figure 2a shows the spatial variation of the PL intensity of sample 1 at the average excitonic peak energy $(\sim 1.906 \mathrm{eV})$ over the scan area. Select locations of higher or lower excitonic peak PL intensity have been marked with black (higher intensity) or white (lower intensity) circles. Figure $2 \mathrm{~b}$ shows the spectra at the two locations in the PL map marked by the leftmost black and white circles superimposed upon each other. It can be seen that the low-energy band and excitonic peak are clearly anti-correlated: regions where the low-energy band is enhanced correspond to a weaker excitonic peak. This scenario repeats itself in comparing the spectra at any pair of locations marked by black and white circles. In fact, while the total integrated intensity does not change significantly between these points, the ratio of the relative strength of the two peaks varies by a factor of 2-3 between the spectra taken at the locations labeled by black/white circles. This relationship implies a competition between the two spectral elements: the low-energy band steals photocarriers from the excitonic transistion.

The strength of the excitionic peak is often cited as a measure of material quality. Conventional thinking contends that large-domain materials show a stronger excitonic peak because of the lower density of domain boundaries [2,3], and it has often been implied that the low-energy band is associated with the domain boundaries. The spatial extent over which the defect-rich regions appear is much larger than the domain size in the material $(\sim 0.3-1.5 \mu \mathrm{m})$. We can thus reject the idea that the low-energy band PL emission originates solely at the domain boundaries, as if this were true, the observed anti-correlation would appear only over small regions which appear between domains. We also can eliminate the possibility that there 
are large domains of disordered material, as the excitonic peak is still present in the defect rich regions. While these facts alone cannot preclude the association of the low-energy band with the domain boundary, they provide evidence that the origin of the low-energy band is not solely associated with the domain boundary and must also originate at other defects within the ordered domain as well.

Results from CdTe sample: Figure 3a shows the spatial variation in the PL intensity from sample 2 at the excitonic peak energy. Select regions are labeled by letters $\mathrm{A}-\mathrm{C}$, the full spectrum taken at each corresponding point is then displayed in Figure 3b. It is our experience that the grain size in these films roughly correlates with the film thickness, which in this case is $\approx 5 \mu \mathrm{m}$, thus we expect to see similarly large grain sizes of the order of $\sim 5 \mu \mathrm{m}$. It is then reasonable to assume that the large dark region in Figure 3 contains a grain boundary. With this assumption, we have plotted the spectra at the select points $\mathrm{A}-\mathrm{C}$, with the intention of revealing the spectral changes which are manifested at the grain boundary.

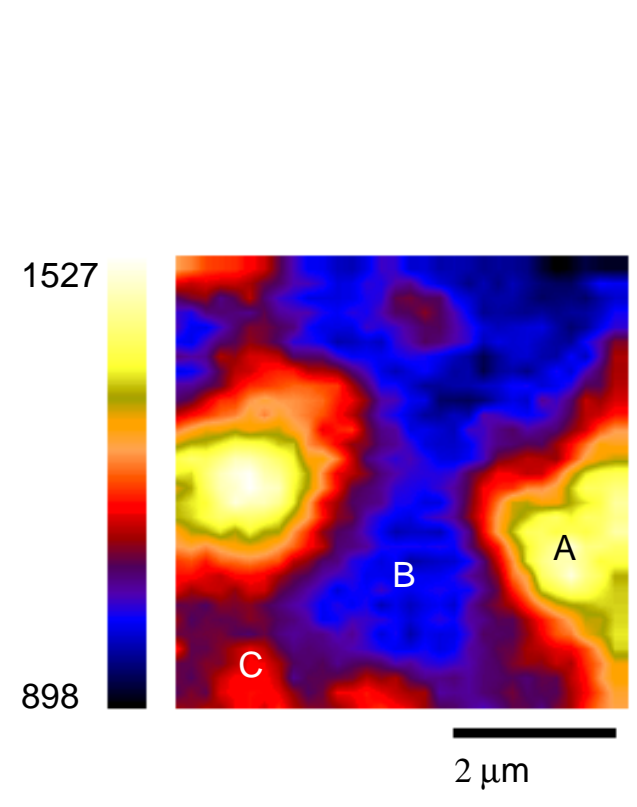

(a)

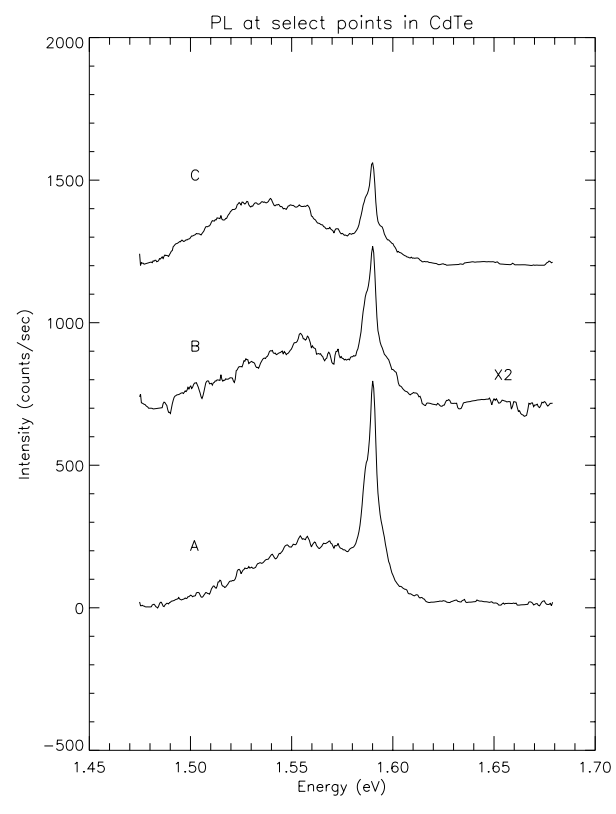

(b)

FIGURE 3. (a): Spatial variation in PL intensity at excitonic peak energy (scale in units of counts/second). (b): Spectra taken at corresponding points A-C in Figure 3a (spectra B \& C offset from 0 for clarity).

In inspecting Figure 3, the following points can be made: 1) the PL intensity varies by a factor of $\approx 2$ as we move from point $A$ to point $B, 2$ ) the broad low-energy PL peak position shifts $\approx 25$ meV from point $\mathrm{A}$ to $\mathrm{C}$, and 3 ) there appear highly localized regions of higher PL intensity ( $\sim 1-2 \mu \mathrm{m}$ spatial extent). Point 1 is consistent with the picture that the grain boundary acts as a non-radiative recombination center, indeed, similar observations were made in our earlier 1-dimensional spatially resolved PL of this system [4]. Point 2 is also consistent with our earlier work and 

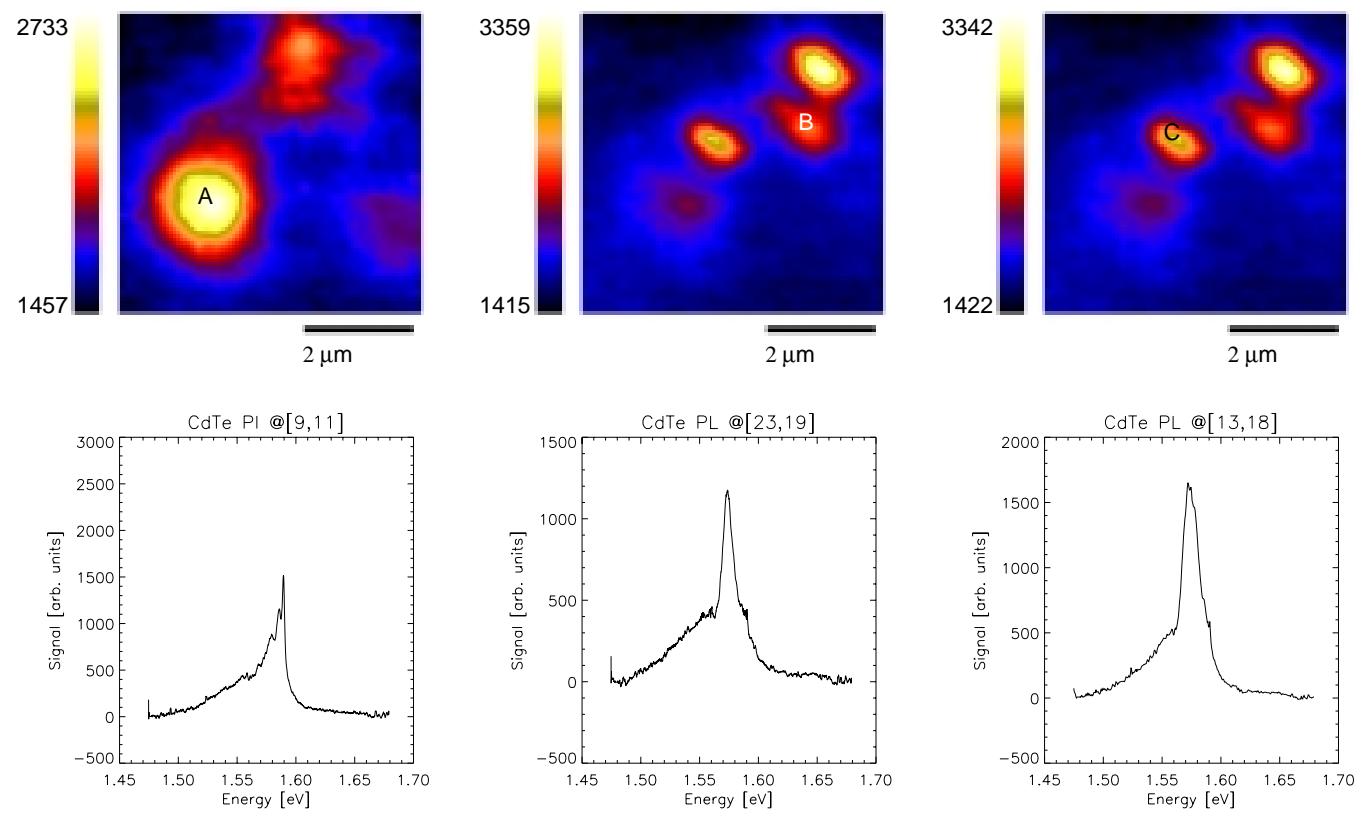

(a)

(b)

(c)

FIGURE 4. (a)-(c): Spatial variation in PL intensity (scale in units of counts/second) at three distinct energies defined by the excitonic peak in the spectra taken at locations labeled A-C in each of the three images (each corresponding spectra shown below image).

can be interpreted in terms of the variation in sulfur alloying due to interdiffusion of sulfur between the $\mathrm{CdS}$ window layer and the CdTe layer. As the peak position is a decreasing function of sulfur content, the area labeled $\mathrm{C}$ can be interpreted as a higher sulfur content grain, and the variation from point $\mathrm{A}$ to $\mathrm{C}$ clearly shows the large variations from grain to grain. Point 3 indicates that an overwhelmingly large portion of the film suffers from relatively poor luminescence and thus a high concentration of non-radiative recombination centers. Only in select portions of the film is relatively higher PL efficiency observed. This phenomenon is repeatedly observed in different areas of the film, as is further illustrated in Figure 4a-c, where we show the PL intensity map at three select energies and the spectra taken at the points labeled A-C (measurements made at a different location in the CdTe sample than those of Figure 3). Each image shows the spatial variation in the PL intensity at the peak energy defined by the corresponding spectra. Again we observe small portions of the film which have an enhanced PL efficiency. The regions may well be localized to different grains, or possibly imperfections within a single grain. The fact that the low-energy peak position is significantly different than that of Figure $3(\approx 10-20 \mathrm{meV}$ shift to higher energy) indicates a relatively lower sulfur concentration (within the current interpretation) in this area of the sample. 


\section{SUMMARY}

In summary, by using high spatial resolution scanning confocal microscopy (demonstrated spatial resolution $\sim 0.7 \mu \mathrm{m}$ ) combined with low-temperature photoluminescence spectroscopy, we demonstrate spatially resolved low-temperature photoluminescence in two photovoltaic materials: a $10 \mu \mathrm{m} \mathrm{GaInP} \mathrm{P}_{2}$ epilayer and a released CdTe thin film. The measured results shed light into the defect-related PL in these systems and its relationship to sub-micron structure. Specifically, we find large areas (greater than the size of a single ordered domain) in the $\mathrm{GaInP}_{2}$ film wherein the low-energy PL is enhanced at the expense of the excitonic peak, and we observe large variations in sulfur interdiffusion and alloying at the CdS/CdTe interface of a released CdTe thin film, as well as small isolated regions of relatively enhanced PL intensity.

\section{ACKNOWLEDGMENTS}

The authors wish to thank Mark W. Keller of NIST, Boulder, CO, for preparing the masked sample. This research was supported by the Offfice of Energy Research (Material Science Division) of the U.S. Department of Energy under Contract No. DE-AC36-83CH10093.

\section{REFERENCES}

1. H.M. Cheong, A. Mascarenhas, J.F. Geisz, J.M. Olson, M.W. Keller and J.R. Wendt, Phys. Rev. B, Rapid Comm. 57, R9400 (1998).

2. P. Ernst, C. Geng, G. Hahn, F. Scholz, H. Schweizer, F. Phillup, and A. Mascarenhas, J. Appl. Phys. 79, 2633 (1996).

3. H.M. Cheong, Y. Zhang, A. Mascarenhas, J.F. Geisz and, J.M. Olson, J. Appl. Phys. 83, $1773(1998)$.

4. R. Dhere, D. Rose, D. Albin, S. Asher, M. Al-Jassim, H. Cheong, A. Swartzlander, H. Moutinho, T. Coutts, and P. Sheldon, Proc., 25th IEEE Photovoltaics Specialist Conference, Washington, D.C. (1996). 